\section{Slippery Nanoworld}

Enrico Gnecco ${ }^{1}$, Anisoara Socoliuc', Ernst Meyer', Alexis Baratoff ${ }^{\prime}$, Roland Bennewitz ${ }^{2}$, Martin Dienwiebel ${ }^{3}$ and Joost Frenken ${ }^{4}$

'National Center of Competence in Research "Nanoscale Science" and University of Basel, Klingelbergstrasse 82, CH-4056 Basel,

Switzerland

${ }^{2}$ McGill University, Rutherford Physics Building, 3600 rue

University, Montréal, Canada

${ }^{3}$ IAVF Antriebstechnik AG, Im Schlehert 32, 76187 Karlsruhe,

Germany

${ }^{4}$ Kamerlingh Onnes Laboratory, Leiden University, P.O. Box 904, 2300 RA Leiden, The Netherlands

Can two solid bodies slide past each other with negligible fric-

tion? Strictly speaking, zero friction would imply that no energy is dissipated in the sliding process. This means, for example, that sliding would not produce sound or thermal waves. The situation is reminiscent of the flow of a fluid without viscosity or of an electric current without resistance. However, one should be aware of the fact that superfluidity and superconductivity are quantum effects, whereas sliding with negligible friction can be explained on a completely classical basis. This phenomenon occurs

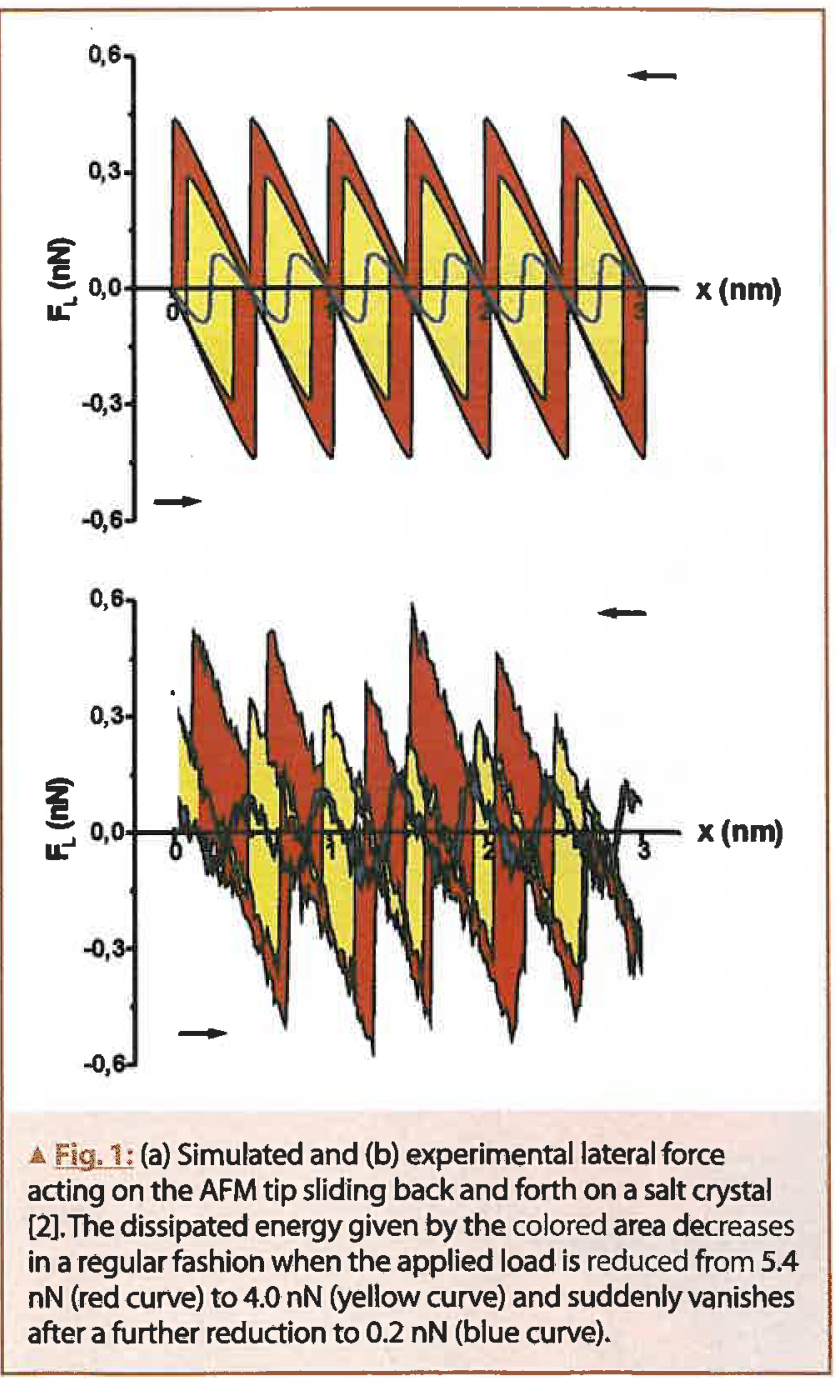

if mechanical instabilities, which otherwise lead to energy dissipation, are suppressed while the bodies are still in contact. Even in that regime an extremely low residual friction must still be present. However, being not related to mechanical instabilities, this residual friction, due to the internal viscosity of the contact, is orders of magnitude lower than conventional dry friction, and to our knowledge falls below the detectability range of any instruments.

In this article we discuss recent experiments which clearly proved the achievement of negligible friction in different physical systems. Two different approaches were exploited to suppress mechanical instabilities. In the first case, negligible friction was observed after reducing the pressure between a sharp tip and a flat surface below a well-defined threshold. In the second case, the cancellation of friction resulted from an incommensurable contact formed between the sliding surfaces.

\section{Transition from stick-slip to continuous sliding in atomic-scale friction}

When two rough surfaces slide past each other, contacts are formed by a complex array of small asperities, which are continually connecting and disconnecting in the sliding process. For the sake of simplicity, we assume that one of the surfaces is perfectly flat and focus on the motion of a single asperity, thus avoiding all complications arising from interactions among asperities mediated by deformations of the sliding surfaces. The simplest model which describes that situation is a sharp tip connected to an external

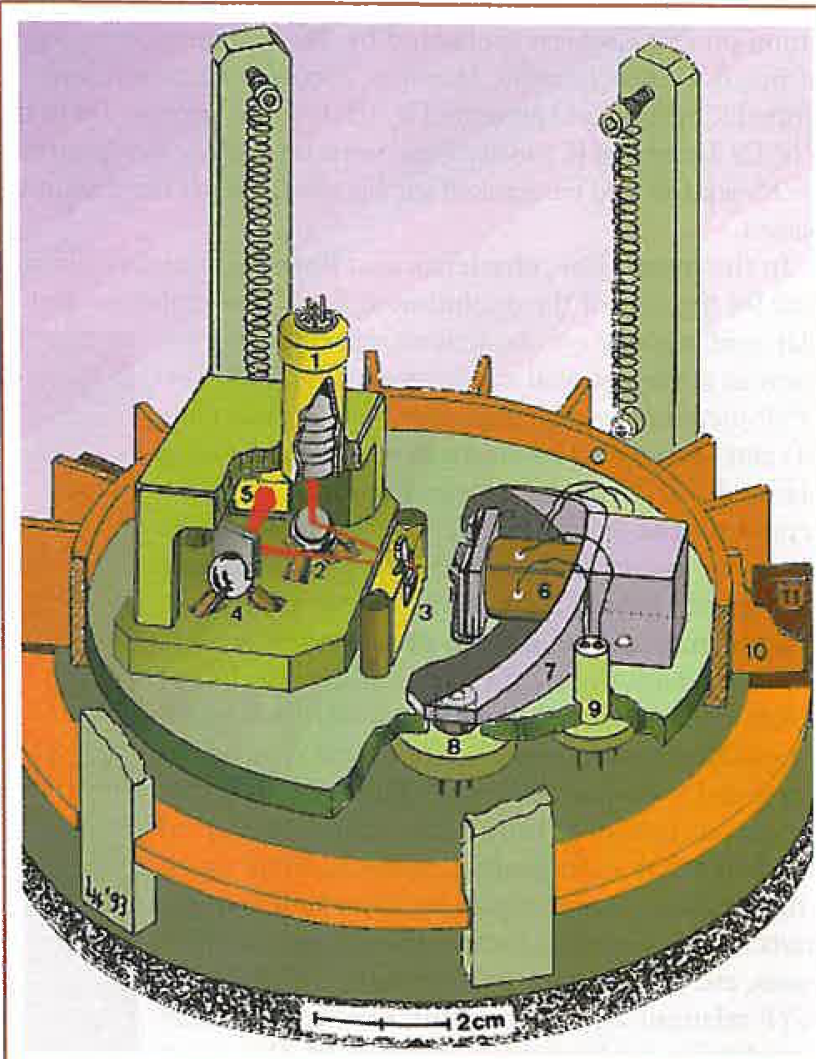

$\triangle$ Fig. 2: The atomic force microscope adopted to detect tiny lateral forces, home-built in Basel: (1) light source with optics; (2), (4) plane mirrors on spherical stepping motors; (3) cantilever holder with probing tip; (5) quadrant photodiode; (6) tube scanner with sample; (7) slider of two-dimensional stepping motor; (8) driving piezo; (9) fixed post; (10), (11) eddy current damping for spring suspension. 
spring which is driven parallel to a surface (Tomlinson, 1929). The surface consists of an array of atoms ordered in a crystal lattice. We also suppose that the tip ends with a single atom, which is not an unrealistic assumption, judging from the resolution of atomicscale features achieved in scanning tunneling and force microscopy. The effect of a larger contact area is discussed in the second part. Under usual conditions, if one tries to pull the spring, the tip remains close to a particular site on the surface until the lateral force becomes large enough to induce a jump into another equilibrium position. This mechanism called atomic stick-slip was first observed by Mathew Mate and coworkers in 1987 [1]. They observed that the positions at which a tungsten tip sliding on graphite sticks, are arranged like the atoms of the surface. Thus, the lattice structure of a surface can be revealed by mapping the lateral force on a tip at the end of a cantilever gently sliding over the surface.

The atomic stick-slip motion is due to the combined action of the elastic force of the spring and of the periodically varying interaction between the tip and the surface. This motion can be recognised via the sawtooth pattern formed when the lateral force is plotted along the sliding path (Fig. 1a, red line). For further comparison with experimental results, the lateral force acquired while scanning backwards is also plotted. Energy is dissipated at each slip, where the position of the tip suddenly becomes unstable and the tip jumps releasing sound and thermal waves into the surrounding material. The total amount of dissipated energy is given by the area enclosed by the friction loop between forward and backward scans The shrill noise of a piece of chalk scratching over a blackboard is generated by the same mechanism, albeit on a much larger length scale. When the pressure on the chalk is reduced the intensity of sound decreases. A lower pressure on the tip corresponds to a lower interaction between tip and surface. The yellow line shows the lateral force expected in such a case. The curves corresponding to the forward and backward scans come closer, so that even though the jumps which reveal instabilities are still present, smaller disjoint friction loops appear and the dissipated energy is reduced. This implies that in each stick stage the equilibrium position of the tip now slightly moves when the spring is extended, due to decreased interaction with the surface. If the pressure on the tip is reduced below a certain threshold, it can be analytically proven that the tip should smoothly follow the spring (blue line). The instantaneous lateral force is not zero, but oscillates between positive and negative values giving rise to zero net dissipation, i.e. zero friction. This picture goes against our common perception of friction, in which the force always opposes the direction of motion. Indeed, the nanoworld behaves differently from the macroworld!

The definite experimental confirmation of this prediction of the Tomlinson model, has come only recently with a sharp hard silicon tip of an atomic force microscope (AFM) gently dragged over a salt crystal surface under dry conditions [2]. In the AFM, the lateral force between tip and surface can be estimated from the torsion of the cantilever that pulls the tip (Fig. 2). The torsion can be detected by a light beam reflected from the cantilever backside. The normal force or load between tip and surface is kept constant during the sliding by controlling the vertical deflection of the cantilever, which is also measured using the same light beam. The experiment was realised under ultra-high vacuum (UHV) conditions, in order to minimise the influence of contaminants. The exceptional signal to noise ratio of the instrument guaranteed the sensitivity required to observe the transition to the regime of negligible friction [3].
Fig. $1 \mathrm{~b}$ shows three friction loops acquired while scanning back and forth a sodium chloride surface cleaved and cleaned in UHV by heating. The red curve represents the lateral force measured with a load of $5.4 \mathrm{nN}$. Each peak corresponds to the tip jumping from one atomic site to the next one. The area enclosed in the loop gives the energy dissipated while crossing six atomic sites. The yellow curve corresponds to a lower load of $4.0 \mathrm{nN}$. The lateral force acting on the tip exhibits split friction loops as in the simulation and the amount of dissipated energy is lower. A further reduction of the load to $0.2 \mathrm{nN}$ has dramatic consequences (blue curve). The tip is now following the cantilever very smoothly, the lateral force is the same in both directions and the energy dissipation becomes negligible. The observed transition occurs while the tip is in contact with the surface. Indeed, a pulling force of 0.7 $\mathrm{nN}$ had to be applied to detach the tip. The quoted loads were estimated by adding this value to the force obtained from the vertical deflection of the cantilever.

\section{Superlubricity of graphite}

Frictionless sliding can also be achieved in a completely different way. When two atomically flat surfaces slide over each other, the

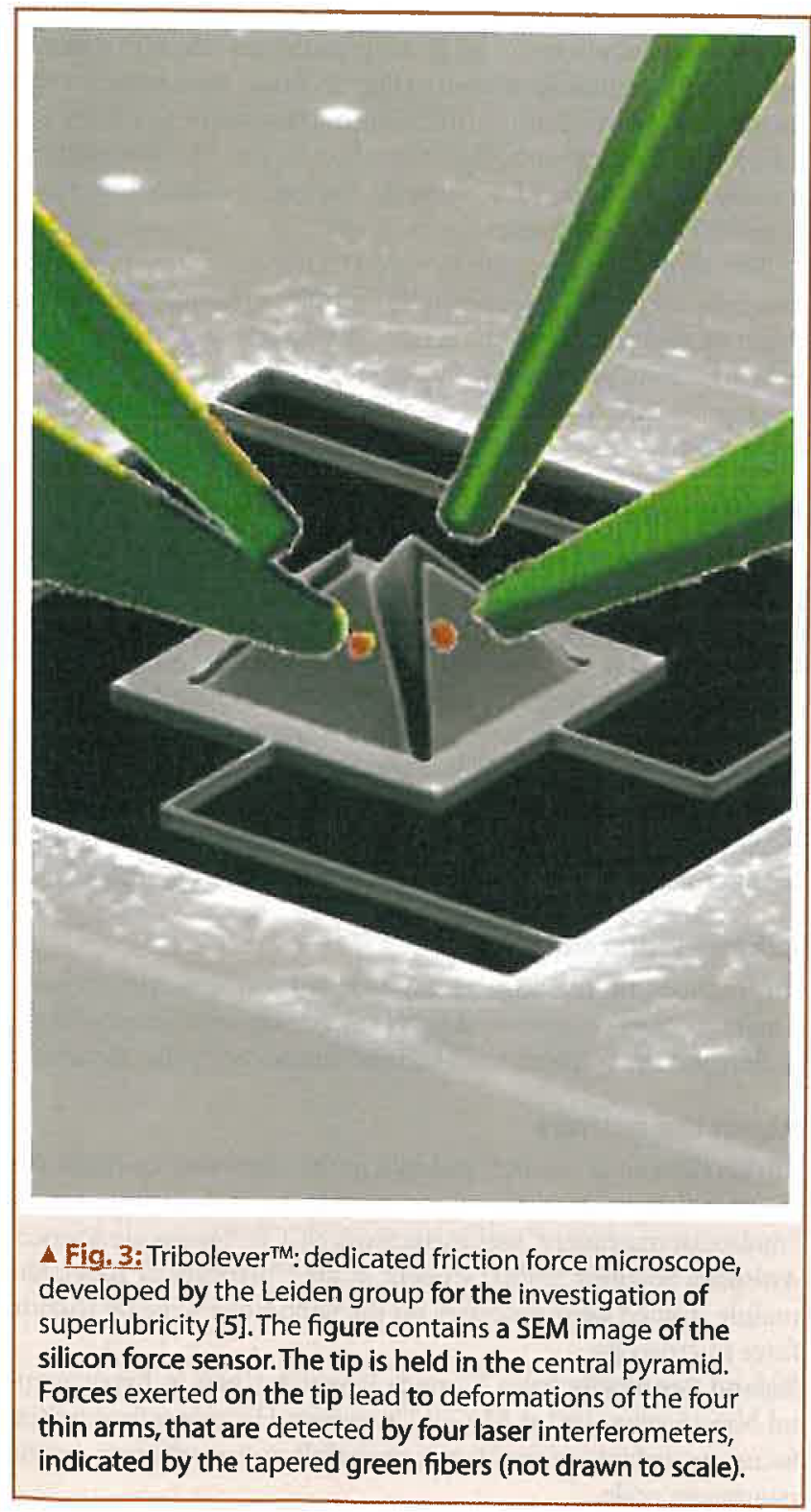


friction can be reduced to zero if (i) the surfaces are in contact over many atoms and (ii) their atomic lattices are incommensurate. This result is due to the fact the lateral forces between the atoms on the two non-fitting surfaces then sum up incoherently. Again, the (combined) lateral force does not reduce to zero but it varies periodically between modest positive and negative values, averaging out to a zero net friction force. In contrast with the previous example of zero friction, the load, pressing the two surfaces together, can be several orders of magnitude above the lateral forces at play inside the contact. This type of extreme friction lowering has been proposed under the name "superlubricity" [4]. Recently, superlubricity has been demonstrated experimentally in a dedicated instrument, the Tribolever (Fig. 3), which allows quantitative tracking of the forces on a scanning tip in three dimensions, with a resolution in lateral forces down to $15 \mathrm{pN}$ [5]. With the Tribolever, a flake from a graphite surface was picked up and the lateral forces between flake and surface were measured at different relative angles of rotation [6]. Stick-slip motion and energy dissipation were clearly visible when the graphite lattices of the flake and the surface were aligned, which occurred at rotation angles of $0^{\circ}$ and $60^{\circ}$. When the flake and the surface were rotated out of registry, to intermediate angles between $0^{\circ}$ and $60^{\circ}$, the friction loops quickly reduced in amplitude, resulting in smooth sliding with negligible friction (Fig. 4). From the rotation angle needed for this reduction in friction, the area of the flake was inferred, in this case roughly 100 carbon atoms. This superlubricity was found to "survive" when the tip was pushed down with appreciable forces of several tens of $\mathrm{nN}$.

The results on graphite have led to the speculation that the excellent lubricating properties of graphite powder may be the result of superlubricity, the motion between lubricated surfaces actually taking place between graphite flakes, most of which are misaligned with respect to each other, therefore sliding with ultralow friction.

\section{Applications}

The observation of negligible friction is a promising result for the practical realisation of nanoelectromechanical systems (NEMS). Despite the increased ratio between surface and volume forces in these devices, an appropriate design of the sliding components should result in smooth motion with minimum energy consumption and without wear of the surfaces in contact. Our results suggest at least two possible ways to achieve this objective: (i) to work at exceptionally low loads and (ii) to exploit incommensurability between surfaces. It is conceivable that these mechanisms are also relevant in the complex mechanics of living organisms.

\section{Acknowledgments}

The authors are thankful Sergey Krylov for fruitful discussions. Their works were supported by the Swiss National Science Foundation and the Netherlands Organisation for Scientific Research.

\section{About the authors}

Enrico Gnecco is research assistant at the University of Basel. His research deals with friction force microscopy and development of "molecular machinery" within the Swiss NCCR "Nanoscale Science". Anisoara Socoliuc is $\mathrm{PhD}$ student at the University of Basel. She mainly studied wear processes on the nanometer scale by friction force microscopy.

Roland Bennewitz has a "Canada Research Chair in Experimental Nanomechanics" at McGill University. His research activities focus on surfaces of insulating materials and mechanics on the nanometer scale.
Ernst Meyer is a Professor of Physics at the University of Basel. His research interests are the development of surface science techniques, such as friction force microscopy and dynamic force microscopy with true atomic resolution.

Alexis Baratoff is Professor of Physics at the University of Basel. His current activities focus on the interpretation and modelling of scanning probe microscopies and of molecular ordering on surfaces. Martin Dienwiebel is now working in the research and tribology group at IAVF Antriebstechnik AG, Germany. His research interests focus on nanoscale wear and friction in lubricated systems. Joost Frenken is a Professor of Physics at Leiden University, where he heads the Interface Physics Group. Central to his research are dynamic aspects of surfaces and interfaces.

\section{References}

[1] C.M. Mate, G.M. McClelland, R. Erlandson, and S. Chiang, Phys. Rev. Lett. 59 (1987) 1942

[2] A. Socoliuc, R. Bennewitz, E. Gnecco, E. Meyer, Phys. Rev. Lett. 92 (2004) 134301

[3] L. Howald, E. Meyer, R. Lüthi, H. Haefke, R. Overney, H. Rudin, and H.-J. Güntherodt, Appl. Phys. Lett. 63 (1993) 117

[4] M. Hirano and K. Shinjo, Phys. Rev. B 41 (1990) 11387

[5] T. Zijlstra, J.A. Heimberg, E. van der Drift, D. Glastra van Loon, M. Dienwiebel, L.E.M. de Groot, J.W.M. Frenken, Sensors and Actuators A: Physical 84 (2000) 18

[6] M. Dienwiebel, G. Verhoeven, N. Pradeep, J. Frenken, J. Heimberg, and H. Zandbergen, Phys. Rev. Lett. 92 (2004) 126101

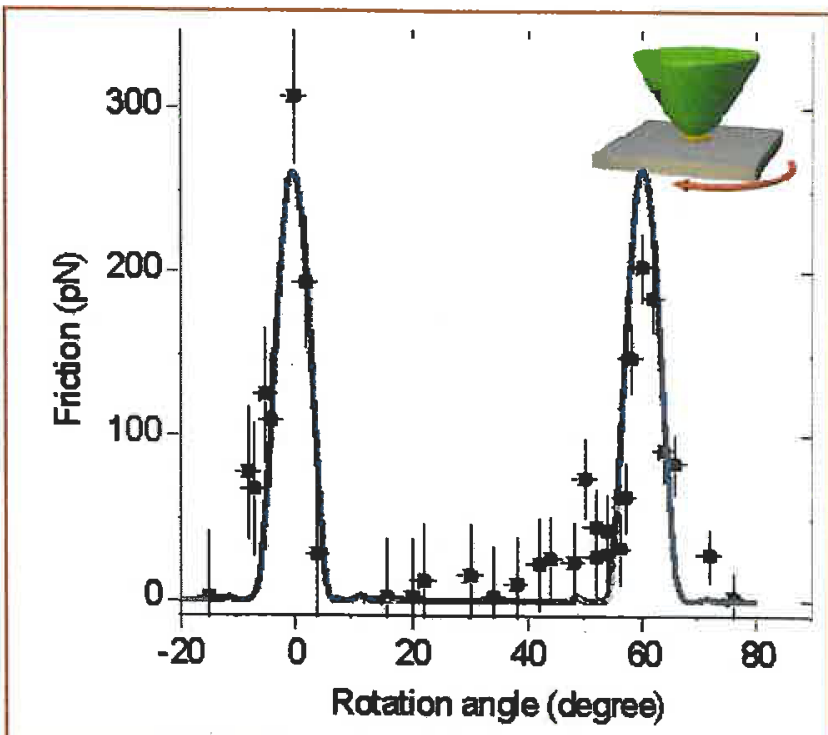

4. Fig. 4: Direct observation of superlubricity of graphite [6]. Each datapoint shows the energy dissipation per unit sliding distance, measured with the Tribolever between a tungsten tip and graphite surface, for one orientation of the tip with respect to the graphite (see inset). These observations are explained by the presence of a graphite flake, picked up by the tip, which slides over the graphite surface. At $0^{\circ}$ and $60^{\circ}$ the lattices of the flake and the substrate match and friction is high. For intermediate angles, the friction is reduced to the detection level of the instrument. The curve is the result of a simple model calculation for a rigid,96-atom graphite flake, sliding over a rigid graphite surface. 